\title{
Measurement of Serum Chitinase 3 Like 1 Protien in Vitiligo Patients
}

\author{
Nagat Gaber Abd ELAziz Hemida ${ }^{b}$, Abd-ElAziz Abd-Elsalam El-Rifaie ${ }^{b}$, Laila Ahmed Rashed $^{a}$, \\ Hanan Abd-Elrazek Kamel $^{b}$ \\ ${ }^{a}$ Biochemistery department, Faculty of Medicine ,Cairo University, Egypt. \\ ${ }^{\mathrm{b}}$ Dermatology and Andrology department, Faculty of Medicine, Beni-Suef University, Egypt.
}

\begin{abstract}
:
The aim of this work was to measure serum level of YKL-40 (CH3L1) protein as an inflammatory biomarker in vitiligo patients. 80 individuals from dermatology outpatient clinic at Beni-Suef University hospital were included in this study; 50 of them are vitiligo cases patients and 30 normal control persons. Blood samples were taken from each participant for measuring of YKL- 40. The serum level of CH3L1 in vitiligo patients was significantly higher as compared to normal control persons ( $\mathrm{p}$-value $<0.001$ ); whereas the mean scores were (107.76 vs. 35.19$)$ in cases and controls respectively.
\end{abstract}

Keywords: vitiligo, Chitinase-3-like protein1, YKL-40.

\section{Introduction}

Vitiligo is one of the acquired, noncontagious diseases that is manifested by progressive multifocal, patchy, depigmented skin associated with or without depigmentation of overlying hair, and mucous membranes due to melanocytes loss in the affected areas. It is non-contagious disease; however, this disorder has sever effect on the psychological behavior of the affected people than their physical capacity more often result in social isolation [1]. Vitiligo is divided into three types and multiple subtypes: Non-segmental, acrofacial, mucosal (involving more than one mucosal area), generalized, universal, mixed (accompanied with segmental vitiligo) and rare variants; Segmental, Undetermined/unclassified vitiligo, focal or mucosal (one site in isolation) [2]. 
In fact, autoimmunity \&oxidative stress in vitiligo patients can stimulate specific systemic manifestations caused by inflammatory \&immunological pathogenesis along with skin involvement. It is thought that an oxidative imbalance accounts for vitiligo development [3]. (Chandhoke et al., 2011) . Chitinase-3 like-1(CH3L1) protein is commonly referred as YKL40 protein. It is a $40-\mathrm{kDa}$ glycoprotein so named for its $3 \mathrm{~N}$ terminal amino acids and its molecular mass as $\mathrm{Y}($ tyrosine $) \& \mathrm{~K}($ lysine $)$, and L(leucine) [4]. Inflammation leads to secretion of this heparin \&chitin-binding lectin without chitinase activity from activated neutrophils \&macrophages in various tissues [5].

In fact, the precise biological role of YKL-40 (CH3L1) isn't clear yet ; but, it might be involved in the inflammation as well as remodeling of the extracellular matrix following injury of tissue [4]. Recently,YKL$40(\mathrm{CH} 3 \mathrm{~L} 1)$ has received a great attention in multiple clinical settings as a potential biomarker in specific pathological conditions which are characterized by tissue inflammation and injury [4].

Elevated serum levels of YLK-40 correlate with morbidity from conditions as rheumatoid arthritis and ongoing liver fibrosis that follow liver injury [6].Besides, high serum concentration of YKL$40(\mathrm{CH} 3 \mathrm{~L} 1)$ is detected in patients suffering from inflammatory bowel
disease(IBD),psoriasis , and giant cell arteritis [7\&8].

Nevertheless, the molecular as well as the cellular mechanisms of these responses are not completely understood yet, and the pathways that connect innate immunity and adaptive immunity during $\mathrm{Th} 2$ responses haven't been adequately determined .Antigen-driven \&antigen-free experimental systems have been used In a trial to detect the mechanisms involved in type 2 responses . Assessment of these systems confirmed the impressive changes in 18 glycosyl hydrolases expression such as the true chitinase acidic mammalian chitinase(AMCase)and the chitinase-like protein(CLP)breast regression protein (BRP)-39/ YKL-40 (CH3L1). These alterations were evaluated in the aeroallergen models of Th2 inflammation\& transgenic mice in which Th2cytokines are overexpressed in a lung- specific manner [9].

This encouraged investigations in order to detect the roles of such molecules in these responses and motivates studies of chitin that is expected to be an important target of some of these moieties. These studies detected that chitinases/ chitinase-like proteins (C/ CLPs) have an essential role in innate along with adaptive type 2 immune responses [10].

During the past decade or so, dysregulation of YKL-40 (CH3L1) has been noted in a wide variety of human disease and disorders characterized by acute 
inflammation, chronic inflammation, and/or tissue remodeling [9].

Although some studies have confirmed the elevated YKL-40 (CH3L1) serum level in patients with psoriasis, [10]. Behcet disease [11]. Atopic dermatitis [12].To our knowledge our study is the first study to measure chitinase 3 like 1 protien in vitiligo patients.

\section{Patients and Methods:}

This study was a case-control study included 2groups: The $1^{\text {st }}$ group included 50 patients with vitiligo. They were males \&females with ages ranging from15 -50 years.

The $2^{\text {nd }}$ group included 30 unaffected individuals as control cases. They were males \&females with ages ranging from 20 -50 years. Patients with vitiligo were recruited from the out-patient clinic of Dermatology Department, Beni-suef University Hospital and within February to July 2019. All patients gave informed consent to participate in this work. The work was approved by the local ethics committee.

\section{Inclusion criteria:}

All patients were enrolled in the study had:

- Different variants and degrees of severity of vitiligo.

\section{Exclusion criteria:}

Any subject was excluded from the study if he/she is:

- Patient with other systemic diseases.

- Patient with other autoimmune disorders as systemic lupus erythematosus,type I D.M, autoimmune thyroid disease, rheumatoid arthritis and Addison's disease .

- Patients proven clinically have chronic inflammatory skin disease as psoriasis and Behcet disease

\section{Methods:}

\section{Specimen collection:}

$-5 \mathrm{ml}$ blood samples were taken from each participant for measuring of YKL- 40

- Samples were collected in serum separator tubes and were allowed to clot for 10-20 min at room temperature before centrifugation for $20 \mathrm{~min}$ at the speed of 2000-3000 r.p.m -serum samples were stored at $-20 \mathrm{c}$.

\section{Statistaic analysis:-}

Analysis of data was done using SPSS (statistical program for social science) version 18 . Frequency distribution with its $\%$ and descriptive statistics with mean \&SD were calculated. Chi-square, t-test, correlations were done whenever needed. P values of $<0.05$ were considered significant. 


\section{Results:}

This study was a case control study on 50 patients with Vitiligo and 30 apparently healthy age and sex- matched induals as controls.

Table (1): Age\& Gender Distribution of Studied Population; (N=80):

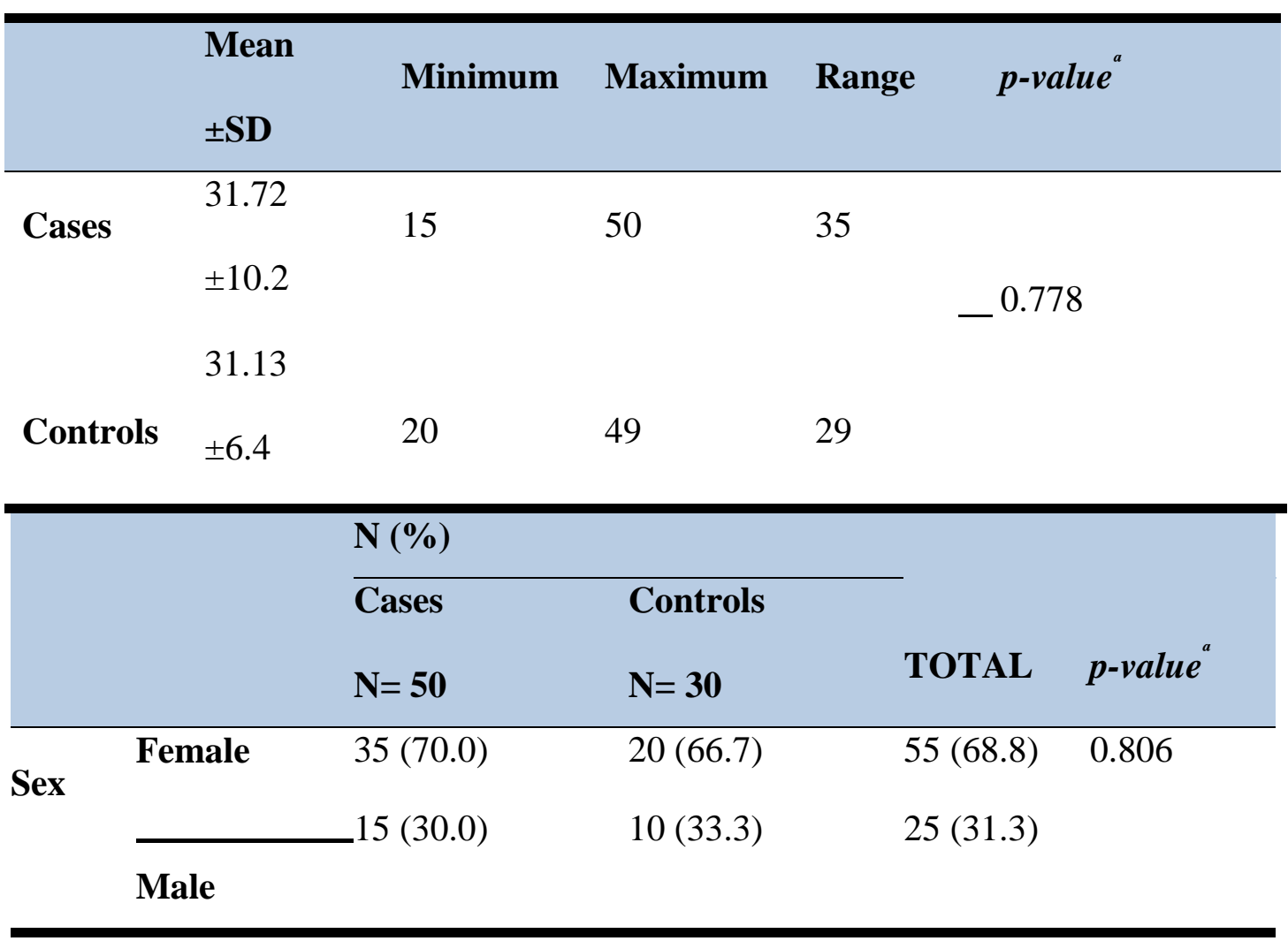

Table (2): Disease Characteristic of studied Vitiligo Cases; $(N=50)$ :

\begin{tabular}{l|l}
\hline \multicolumn{2}{|l}{ Duration of Vitiligo Disease (years) } \\
\hline Mean \pm SD & $4.62 \pm 6.2$ \\
\hline Range (Minimum-Maximum) & $34.9(35-0.1)$ \\
\hline Duration of Last Lesion (months) \\
\hline Mean \pm SD & $5.70 \pm 3.9$ \\
\hline Range (Minimum-Maximum) & $1(12-1)$ \\
\hline
\end{tabular}


Figure (1): Distribution of Vitiligo Lesions in the studied Cases

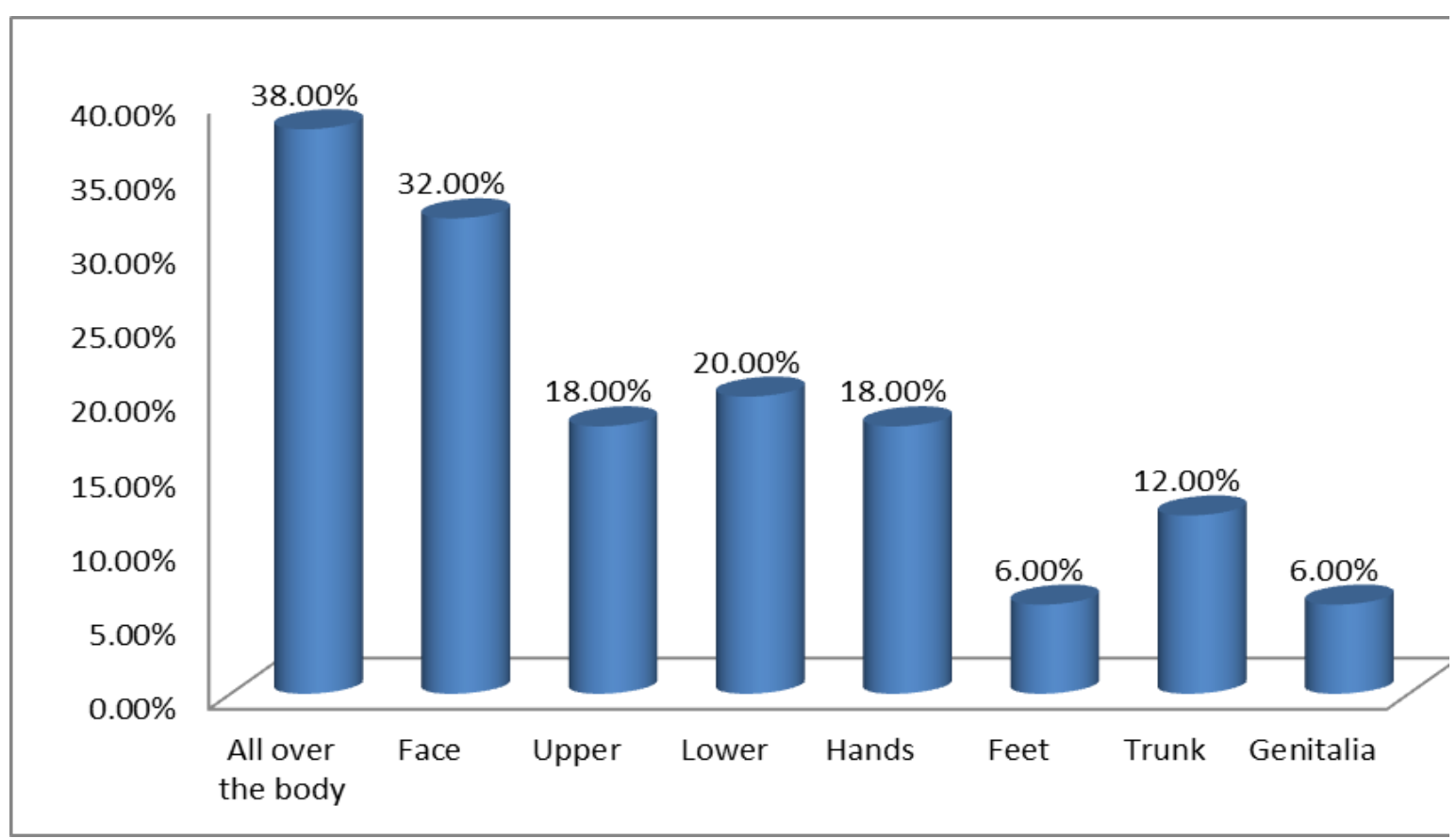

Table (3): The Serum Level of Chitinase 3 like 1(CH3L1) protein in Vitiligo Patients as Compared to Normal Control Persons; $(\mathrm{N}=\mathbf{8 0})$ :

\begin{tabular}{|c|c|c|c|}
\hline & $\begin{array}{l}\text { Cases } \\
\mathbf{N}=\mathbf{5 0}\end{array}$ & $\begin{array}{l}\text { Controls } \\
\mathrm{N}=\mathbf{3 0}\end{array}$ & p-value \\
\hline Mean \pm SD & $107.76 \pm 17.3$ & $35.19 \pm 10.5$ & \multirow[t]{4}{*}{$<0.001 *$} \\
\hline Range & 79.40 & 55.50 & \\
\hline Minimum & 72.70 & 20.70 & \\
\hline Maximum & 152.10 & 76.20 & \\
\hline
\end{tabular}

Table (4): Correlation between The Serum Level of CH3L1 protein in Vitiligo Patients and Age of the studied cases; $(\mathrm{N}=30)$ :

\begin{tabular}{|c|c|c|c|}
\hline & \multicolumn{3}{|c|}{ Age of the studied cases } \\
\hline $\begin{array}{l}\text { Chitinase-3 } \\
\text { protein }\end{array}$ & like-1(CH3L1) & $r=0.042$ & $p$-value $=0.712$ \\
\hline
\end{tabular}


Table (5): Relation between The Serum Level of CH3L1 protein in Vitiligo

Patients and sex distribution of the studied cases; $(\mathrm{N}=30)$ :

\begin{tabular}{llll} 
& Females & Males & \multirow{2}{*}{ P-value } \\
\cline { 1 - 2 } & $\mathbf{N}=\mathbf{3 5}$ & $\mathbf{N}=\mathbf{1 5}$ & \\
\cline { 1 - 3 } Mean \pm SD & $109.36 \pm 15.8$ & $104.03 \pm 20.3$ & \\
\cline { 1 - 2 } Minimum & 75.70 & 72.70 & \\
\cline { 1 - 2 } Maximum & 129.40 & 152.10 & \\
\cline { 1 - 2 } Range & 53.70 & 79.40 & \\
\hline
\end{tabular}

Table (6): Correlation between The Serum Level of CH3L1protein in Vitiligo Patients and Disease Duration of the studied cases; $(\mathrm{N}=50)$ :

\begin{tabular}{lllr}
\hline & \multicolumn{2}{c}{ Disease Duration (years) } \\
\hline Chitinase-3 & like-1(CH3L1) & $r=0.284$ & $p$-value $=\mathbf{0 . 0 4 5} *$ \\
protein & & & \\
\hline
\end{tabular}

Table (7): Correlation between The Serum Level of CH3L1 protein in Vitiligo Patients and Duration of last lesion of the studied cases; $(N=30)$ :

\begin{tabular}{|c|c|c|}
\hline & & Duration of last lesion (months) \\
\hline $\begin{array}{l}\text { Chitinase-3 } \\
\text { protein }\end{array}$ & like-1(CH3L1) & $p$-value $=0.941$ \\
\hline
\end{tabular}

Table (8): Relation between The Serum Level of CH3L1 protein in Vitiligo Patients and Family History of the studied cases; $(\mathrm{N}=50)$ :

\begin{tabular}{llll}
\hline & Negative & Positive & \multirow{2}{*}{ P-value } \\
& $\mathbf{N = 3 1}$ & $\mathbf{N = 1 9}$ & \\
\hline Mean \pm SD & $109.73 \pm 16.5$ & $104.54 \pm 18.5$ & 0.789 \\
\cline { 1 - 2 } Minimum & 72.70 & 70.20 & \\
\hline
\end{tabular}




\begin{tabular}{lll}
\hline Maximum & 152.10 & 129.30 \\
\hline Range & 79.40 & 59.10
\end{tabular}

\section{Discussion:}

Vitiligo is one of the acquired diseases that have a variable course. Clinically, it is characterized by the presence of well-defined depigmented patches or macules thought to result from melanocyte dysfunction loss. It is the commonest depigmentation disorder that affect about $0.5-2.0 \%$ of the population without any predilection for gender or race [13].

In vitiligo theory of innate immunity, synthesis as well as release of elevated level of pro-inflammatory proteins\& cytokines such as HSP, IL-1 $\beta$, IL6, and IL-8 was proved [14].CHI3L1has been recommended to have a critical role in a lot of procedures as inflammation, migration, and proliferation of malignant cells, angiogenesis, tissue remodeling [14].

The serum level of $\mathrm{CH} 3 \mathrm{~L} 1$ in vitiligo patients was significantly higher as compared to normal control persons ( $\mathrm{p}$-value $<0.001$ ); whereas the mean scores were(107.76 vs. 35.19) in cases and controls respectively

In a study for pathogensis of vitiligo, autoimmune theory is accepted and this is confirmed by the frequent association of vitiligo with diseases having an autoimmune origin such as Graves' disease, Hashimoto's thyroiditis, T1DM, and Addison's disease. As cytokines are essential mediators of immunity, there are evidence that suggested that they have a pivotal role in autoimmune disease pathogenesis [15].

In the same study, serum IL-6\&IL-2 levels in the patient group were measured and they were significantly higher in comparison with those of the normal controls and this might have a critical role in melanocytic cytotoxicity. So, we speculate that the cytokine production of epidermal microenvironment could be incorporated in vitiligo [15].

Vitiligo is usually accompanied by other autoimmune disorders. In a recent survey of $>2600$ unselected Caucasian vitiligo patients, increased frequencies of autoimmune thyroid disease, SLE, Addison's disease, and pernicious anemia were documented, with nearly $30 \%$ of patients being suffering from at least one additional autoimmune disorder [16].

In a recent study that assessed vitiligo and its association with other autoimmune disorders, thyroid disease was the most frequent one followed by psoriasis, DM and alopecia areata [17].

As mentioned earlier in vitiligo theory of innate immunity, synthesis as well as 
release of elevated level of pro-inflammatory proteins\& cytokines such as HSP, IL-1 $\beta$, IL6, and IL-8 was proved.

CHI3L1or YKL- 40 or BRP-39 or HC gp-39, is a chitinases like protein that has been established in human [9].

In 1993, it was cloned and described as an important secretory product of chondrocyte and fibroblast cells of synovium from patients with rheumatoid arthritis [18].

CHI3L1has been recommended to have a critical role in a lot of procedures as inflammation, migration, and proliferation of malignant cells, angiogenesis, tissue remodeling [19].

The biological function of YKL-40 is still unclear; however, many possible functions have been proposed; [20].documented in an in vitro study that YKL-40 enhance the rate of growth of 3 fibroblastic cell lines derived from human osteoarthritic synovium, fetal lung and adult skin [21]. IL-6 stimulates YKL-40 synthesis in human in vivo studies [21].

In a study for Aydın, Tekirdag, 2015 Instit, Namık Kemal University of Health Sciences, Department of Medical Biochemistry Postgraduate Thesis for assessing Factor YKL-40 level in Patients with Alopecia Areata.Patient's serum YKL40 levels were elevated than control group'sYKL-40 level [22].

In a study measuring the plasma levels of YKL-40 and its association with malondialdehyde (MDA)in Behcet's disease patients, results revealed non-significant correlation between it and MDA ( $p>0.05$ ), and concluded that CHI3L1may be associated with Behcet's disease. Elevated MDA level wasn't only the etiology of inflammation, but also it was an indicator of oxidative stress in Behcet's disease [23].

In a new study for determination of CHI3L1as a neutrophil antigenic target in Crohn's and coeliac disease using ELIZA and proved that CHI3L1was elevated than normal and higher in Crohn's disease than in Coeliac and ulcerative colitis [24].

The recent our study is a case-control study aimed to measure the level of CH3L1 in 50 vitiligo cases patients and comparing it to 30 normal control persons to determine the level of CH3L1in the disease.

This study includes 80 individuals from dermatology outpatient clinic at Beni-Suef University hospital during the period (from 1

\section{February 2019 to 1 July 2019).}

Our study is the first study to measure chitinase 3 like 1 protein in vitiligo patients. The individuals were grouped into two groups matched in age and sex distribution as 50 individuals suffering from vitiligo in one group and 30 healthy controls in another group.

The serum level of $\mathrm{CH} 3 \mathrm{~L} 1$ in vitiligo patients was significantly higher as compared to normal control persons ( $\mathrm{p}$-value $<0.001$ ); 
whereas the mean scores were (107.76 vs. 35.19) in cases and controls respectively.

\section{References:}

1- Laddha NC, Dwivedi M, Begum R. Increased tumor necrosis factor (TNF)- $\alpha$ and its promoter polymorphisms correlate with disease progression and higher susceptibility towards vitiligo. PLoS ONE 2012; 7 :e52298.

2- Ezzedine, K., Lim, H. W., Suzuki, T., Katayama, I., Hamzavi, I., Lan, C. C. E., Goh, B. K., Anbar, T., Silva de Castro, C., Lee, A. Y.,Vitiligo Global Issue Consensus Conference Panelists. (2012). Revised classification/nomenclature of vitiligo and related issues: the Vitiligo Global Issues Consensus Conference. Pigment Cell \&Melanoma Research 25(3), E1E13.doi:10.1111/j.1755- 148X.2012.00997.x

3- Roberta Colucci, Federica Dragoni, Silvia Moretti, "Oxidative Stress and Immune System in Vitiligo and Thyroid Diseases", Oxidative Medicine and Cellular Longevity, vol. 2015, Article

ID 631927, 7 pages, 2015. https://doi.org/10. $1155 / 2015 / 631927$

4- Kastrup, J. (2012). Can YKL-40 be a new inflammatory biomarker in cardiovascular disease? Immunobiology, 217(5), 483-491. doi:10.1016/j.imbio.2011.04.007

5-Simsek, I., Meric, C., Erdem, H., Pay, S., Kilic, S., \& Dinc, A. (2008). Accuracy of recall of the items included in disease activity forms of Behçet's disease: comparison of retrospective questionnaires with a daily telephone interview. Clinical Rheumatology, 27(10), 1255- 1260. doi:10.1007/s10067008-0899-8

6- Rath, T., Roderfeld, M., Güler, C., Wenzel, C., Graf, J., Beitinger, F., Roeb, E., \& Zachoval, R. (2011). YKL-40 and transient elastography, a powerful team to assess hepatic fibrosis. Scandinavian Journal of Gastroenterology, 46(11), 1369-1380. doi:10.3109/00365521.2011.613949

7- Ahmed, S. F., Attia, E. A. S., Saad, A. A., Sharara, M., Fawzy, H., \& El Nahrery, E. M. A. (2015). Serum YKL-40 in psoriasis with and without arthritis; correlation with disease activity and high-resolution power Doppler ultrasonographic joint findings. Journal of the European Academy of Dermatology and Venereology, 29(4), 682-688. doi:10.1111/jdv.12653

8- Erfan, G., Guzel, S., Alpsoy, S., Rifaioglu, E. N., Kaya, S., Kucukyalcın, V., Topcu, B., \& Kulac, M. (2015). Serum YKL-40: a potential biomarker for psoriasis or endothelial dysfunction in psoriasis? Molecular and Cellular Biochemistry, 400(1-2), 207-212. doi:10.1007/s11010-014-2277-y

9- Lee C.G, C. A. Da Silva, C. S. Dela Cruz et al.(2011): "Role of chitin and chitinase/chitinase-like proteins in inflammation, tissue remodeling and injury," 
Annual Review of Physiology, vol. 73, pp. $479-501$.

10-Hartl, D., Lee, C. G., Da Silva, C. A., Chupp, G. L., \& Elias, J. A. (2009). Novel biomarkers in asthma: chemokines and chitinase-like proteins. Current Opinion in Allergy and Clinical Immunology, 9(1), 6066. doi:10.1097/ACI.0b013e32831f8ee0

11-Saba, M., Sharif, M. R., Akbari, H., Nikoueinejad, H., \& Ramazani Jolfaii, M. (2014). YKL-40 in Asthma and its correlation with different clinical parameters. Iranian Journal of Allergy, Asthma, and Immunology,13(4),271-7. Retrieved from http://www.ncbi.nlm.nih.gov/pubmed/246591 $\underline{63}$

12- Werfel, T., Allam, J.-P., Biedermann, T., Eyerich, K., Gilles, S., Guttman-Yassky, E., Hoetzenecker, W., Knol, E., Simon, H.-U., Wollenberg, A., ... Akdis, C. A. (2016). Cellular and molecular immunologic mechanisms in patients with atopic dermatitis. Journal of Allergy and Clinical Immunology, 138(2), 336-349. doi:10.1016/j.jaci.2016.06.010

13-Ezzedine, Khaled, Eleftheriadou, V., Whitton, M., \& van Geel, N. (2015). Vitiligo. The Lancet, 386(9988), 74-84. doi:10.1016/S0140- 6736(14)60763-7

14-Rashighi, Mehdi, \& Harris, J. E. (2017). Vitiligo Pathogenesis and Emerging Treatments. Dermatologic Clinics, 35(2), 257-265. doi:10.1016/j.det.2016.11.014
15-Singh, S., Khandpur, S., Sharma, V. K., \& Ramam, M. (2013). Comparison of efficacy and side-effect profile of oral PUVA vs. oral PUVA sol in the treatment of vitiligo: a 36week prospective study. Journal of the European Academy of Dermatology and Venereology, 27(11), 1344-1351. doi:10.1111/jdv.12002

16-Mollet, I., van Geel, N., \& Lambert, J. (2010). Autoimmune/inflammatory and other diseases associated with vitiligo. In Vitiligo (pp. 79-90). Springer, Berlin, Heidelberg.

17- van den Boorn, J. G., Konijnenberg, D., Dellemijn, T. A. M., Wietze van der Veen, J. P., Bos, J. D., Melief, C. J. M., Vyth-Dreese, F. A., \& Luiten, R. M. (2009). Autoimmune Destruction of Skin Melanocytes by Perilesional T Cells from Vitiligo Patients. Journal of Investigative Dermatology, 129(9),2220-2232. doi:10.1038/jid.2009.32

18- Hakala B.E, C. White, and A. D. Recklies(2000): "Human cartilage gp-39, a major secretory product of articular chondrocytes and synovial cells, is a mammalian member of a chitinase protein family," Journal of Biological Chemistry, vol. 268, no. 34, pp.2581-25803.

19- Jefri M, Huang Y-N, Huang W-C, Tai C-S, Chen W-L (2015): YKL-40 regulated epithelial-mesenchymal transition and migration/invasion enhancement in non-small cell lung cancer. BMC Cancer .15:59 
20- Kazakova, M. H., \& Sarafian, V. S. (2017). YKL-40: The Search for New Biomarkers in Rheumatoid Arthritis. New Developments in the Pathogenesis of Rheumatoid Arthritis, 139.

21- Johansen JS, Christoffersen P, Moller S, Price PA, Henriksen JH, et al. (2000): Serum YKL-40 is increased in patients with hepatic fibrosis. J Hepatol 32:911-920 .

22- Aydin, B. (2015). Alopesi areata'lı (AA) hastalarda serum YKL-40 ve TGF- $\beta 1$ düzeyinin değerlendirilmesi (Master's thesis, Namık Kemal Üniversitesi)
23- Bilen, H., Altinkaynak, K., Sebin, E., Aksoy, H., \& Akcay, F. (2016). Serum YKL40 and MDA levels in Behçet disease. J Pak Med Assoc, 66(10), 1299-30

24- Claudia Deutschmann, Mandy Sowa, Jayaseelan Murugaiyan, Uwe Roesler, et al Identification of Chitinase-3-Like Protein 1 as a Novel Neutrophil Antigenic Target in Crohn's Disease, Journal of Crohn's and Colitis, Volume 13, Issue 7, July 2019, Pages 894-904, https://doi.org/10.1093/eccojcc/jjz01 